\title{
Experimental benchmark data for PWR rod bundle with spacer-grids
}

\author{
Elvis E. Dominguez-Ontiveros ${ }^{1}$, Yassin A. Hassan ${ }^{1}$ \\ Michael E. Conner ${ }^{2}$, Zeses Karoutas ${ }^{2}$ \\ ${ }^{I}$ Nuclear Engineering Department, Texas A\&M University, College Station, TX, USA 77843- \\ 3133 \\ ${ }^{2}$ Westinghouse Nuclear Fuel, 5801 Bluff Road, Columbia, SC, USA 29209
}

\section{Introduction}

Fluid flow around circular cylinders is one of the classical problems of fluid mechanics and has been well studied because of its common occurrence in many forms and in different applications. Cylinder-like structures can be found both alone and in groups in the designs for heat exchangers, cooling systems for nuclear power plants, offshore structures, power lines, struts, grids, screens, and cables, in both single and multi-phase flows. A complete understanding of the fluid dynamics for the flow around a circular cylinder includes such fundamental subjects as the boundary layer separation, the free shear layer, the wake, and the dynamics of vortices. The flow fields of multiple-cylinder configurations involve complex interactions between the shear layers, vortices and Karman vortex streets (Zdravkovich 1987). The problem is further complicated by the large number of configurations encountered in practice, resulting in different flow patterns, and by the effect of their interactions. One of the applications of paramount importance in this study is fluid flow in fuel rod bundles of Light Water Reactors (LWR). In this type of nuclear reactor, optimum heat removal from the surface of fuel elements is the subject of many studies for researchers in order to determine reactor thermal margin and safety. In this case, the spacer grids which support the fuel assembly are used as an effective mixing device by attaching various types of flow deflectors. Several recent works focused on the development of numerical simulations that predict the complex behavior of fluid flow close to grid spacers and between fuel assemblies ( Ikeno 2006, Caraghiaur 2007). However, the validity of the produced results from Computational Fluid Dynamics (CFD) is still under scrutiny for several cases in real world conditions. Moreover, the existing models for multiphase flows produce results that are generally recognized as unreliable. Development of better models for multiphase simulation requires an improved understanding of the evolution of the flow with dynamic interaction of the flow multi-scales. Therefore, experimental data is needed for thorough validation of the models.

In numerical simulations of fuel rod bundle flow fields, the unsteady Navier-Stokes equations have to be solved in order to determine the time (phase) dependent characteristics of the flow. In order to validate the simulations results, detailed comparison with experimental data must be done. Experiments investigating complex flows in rod bundles with spacer grids that have mixing devices (such as flow mixing vanes) have mostly been performed using single-point measurements by traversing between the rod gaps and within the rods passages. A variety of probes has been used in these investigations including, hot-wire (Rehme 1987), hot-film, LDV (Neti 1982), five-hole pitot tube, high response pressure transducer, etc. Although these measurements allow local comparisons of experimental and numerical data (e.g. line distributions of velocity components and/or Reynolds stresses in the vicinity of the mixing vanes, passages, rod gaps, etc.), they provide little insight because the discrepancies can be due to the integrated effects of many complex flow phenomena such as wake-wake, wake-vane, and vane-boundary layer interactions occurring simultaneously in a complex flow environment. The 
same issue exists for full lateral flow field data from PIV measurements in the open literature; most of the existing data provide average flow data not time dependent.

In order to obtain more details and insight on the discrepancies between experimental and numerical data as well as to obtain a global understanding of the causes of these discrepancies, comparisons of the distributions of complete phase-averaged velocity and turbulence fields for various locations near spacer-grids should be performed. The experimental technique Particle Image Velocimetry (PIV) is capable of providing such benchmark data utilizing current PIV equipment and computers. However, PIV requires optical access for the laser sheet and the camera view to the region of interest, whereas the flow field in a typical fuel bundle with spacer-grids is usually optically obstructed by the rods themselves. In addition, light reflections from the rod surface and end walls tremendously affect the quality of images, particularly near the boundaries. As a result, previously obtained data in rod bundles have covered limited areas, close to the boundaries and mostly in small sections of the bundle (Neti 1982, Ikeda 2006, Caraghiaur 2007). Most of these studies are also performed with instrumentation that provides either spatial or temporal resolution of the acquired data and are not suitable for acting as a detailed benchmark dataset for rod bundle with spacer grids flow computations.

This paper describes an experimental database obtained using two-dimensional Time Resolved Particle Image Velocimetry (TR-PIV) measurements within a 5 x 5 PWR rod bundle with spacer-grids that have flow mixing vanes. One of the unique characteristic of this set-up is the use of the Matched Index of Refraction technique employed in this investigation which consists of immersing plastic rods with a similar index of refraction as the one for water to achieve optical transparency zones in the neighborhoods of the spacer grids. This unique feature allows flow visualization and measurement within the bundle without rod obstruction. This approach also allows the use of high temporal and spatial non-intrusive dynamic measurement techniques namely TR-PIV to investigate the flow evolution below and immediately above the spacer. The experimental data to be presented in this paper includes explanation of the various cases tested such as test rig dimensions, measurement zones, the test equipment and the boundary conditions in order to provide appropriate data for comparison with Computational Fluid Dynamics (CFD) simulations. Turbulence parameters of the obtained data are analyzed in order to gain insight of the physical phenomena. Measurement uncertainties are quantified and the error analysis methodology is presented.

\section{Experimental details}

\subsection{Facility}

The experiments were performed at the Optical Multi-phase Flow Research Laboratory in the Nuclear Engineering Department of Texas A\&M University. The experimental facility consisted of two main systems: the hydraulic loop and the test section as shown in figure 1. Both systems were coupled together in order to obtain flow representations of the studied phenomena. The closed loop configuration consisted of a plastic reservoir tank with capacity for 570 liters, a 2 HP centrifugal pump (Berkeley model: S39538) with modified o-rings for high temperature operation, CPVC valves, filters, and CPVC piping, and various pressure gages. The tank was located $25.4 \mathrm{~cm}$ above the pump intake in order to provide a constant water head to the pump during normal operation. The fluid passed through a series of filters (Keystone filter division model: MMTGB20) that limit the size of suspended particles in the fluid during experiments to $50 \mathrm{e}-6 \mathrm{~m}$. The pump was controlled by a variable frequency controller (Polyspeed Electronics 
Dallas Texas model: XLT25-20C) in order to assure reproducibility of flow rates and constant torque in the pump impeller at various operation frequencies. An insertion propeller type flow meter (Data Industrial SDI flow sensor model: SDI 1D1N10-0200) was located downstream at a distance of $228.6 \mathrm{~cm}$ from the pump's exit to measure the volumetric flow rate delivered to the test section. An air mixing chamber was included in the design of the hydraulic loop in order to inject gas into the main flow stream. Data for gas injection is not presented in this paper.

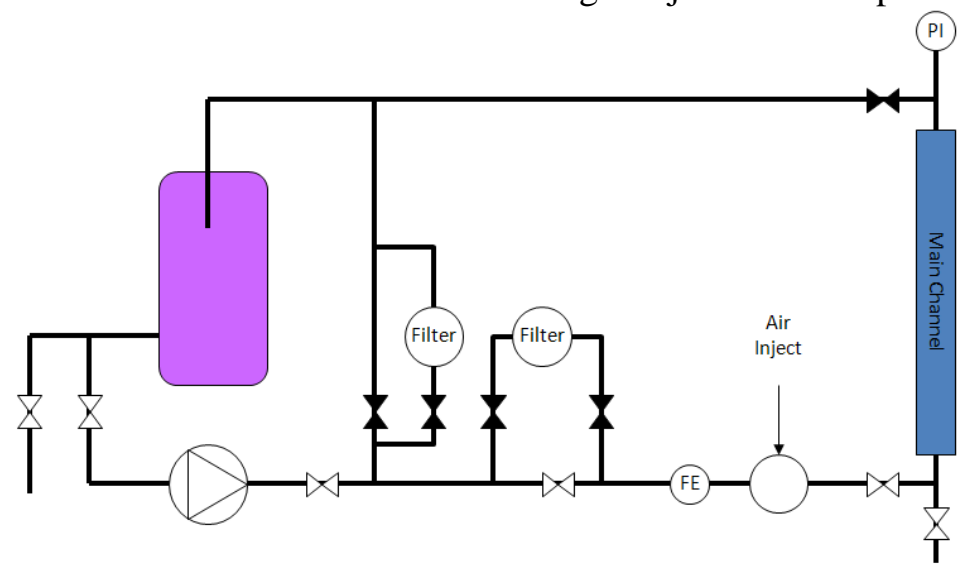

FIGURE 1. Hydraulic loop.

\subsection{Test section}

The test section was formed by an aluminum frame and an inside polycarbonate channel. However, for practical purposes the inside polycarbonate channel was considered as the flow envelope for the rod bundle with spacer grids.The frame was an aluminum rectangular box shaped channel of length $1790.7 \mathrm{~mm}$ and a square cross section with a side length of $122.9 \mathrm{~mm}$. The channel had a total of four (4) rectangular windows on each of the longest faces of the box (one window per side) which served as observation windows.

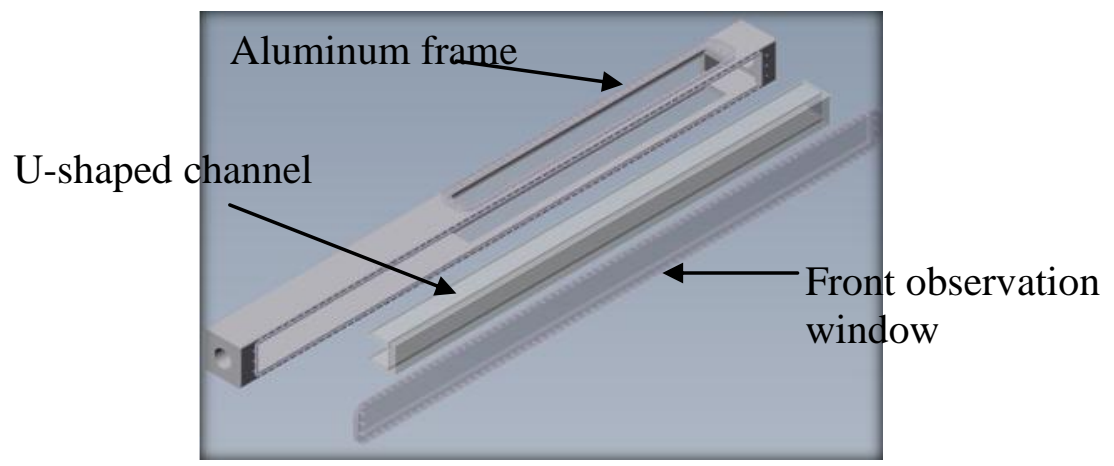

FIGURE 2. Exploded view of aluminum frame, U-shaped channel and front observation window.

A second polycarbonate channel was placed inside the sealed windowed aluminum frame in order to have an even cross section and wall smoothness inside the test section. Therefore by placing a u-shaped channel inside the main channel, it assures the free flow of the fluid and a constant smoothness along the test section. These approaches were considered 
important details taken into account that may avoid geometry problems in the Computational Fluid Dynamics (CFD) simulations by providing a simple geometry model that accurately represents the real geometry in the channel without overlooking significant details. Figure 2 shows an exploded view of the aluminum frame, u-shaped plastic channel and the observation window.

\subsection{Grid}

The spacer grid used for these experiments models a Westinghouse support grid design which is used in actual nuclear fuel assemblies for Pressurized Water Reactors (PWR). The Westinghouse grid design is characterized by an egg-crate shape with mixing vanes located on the downstream edge of the grid in a split vane pair configuration. In this study, a $5 \times 5$ subsection of the real spacer grid geometry was used as the test grid. With the exception of the smaller 5x5 array, the test grid geometry is identical to the actual grid with regards to dimensions; for example grid height, fuel rod pitch $(\mathrm{P}=12.6 \mathrm{~mm})$, fuel rod $\mathrm{OD}(9.5 \mathrm{~mm})$, mixing vane design, and support feature design (springs and dimples). The test grid contains 25 eggcrate spaces in a configuration of $5 \times 5$ rods as shown in figure 3 . The rods were fabricated using a fluorinated ethylene-propylene (FEP) plastic with dimensions of 9.5 OD x 9 ID x 1270 $\mathrm{mm}$ long with a +-0.0762 $\mathrm{mm}$ tolerance in all dimensions. The test rods were closed at each end using a coned aluminum caps and filled with a solution of water and chlorine in a 5\% by volume concentration. This solution allowed keeping the refractive index of water close to its original value of 1.33 and the chlorine restricted the growth of any kind of bacteria or algae in the fluid which may alter the optical transparency of the stagnant solution inside the rods.

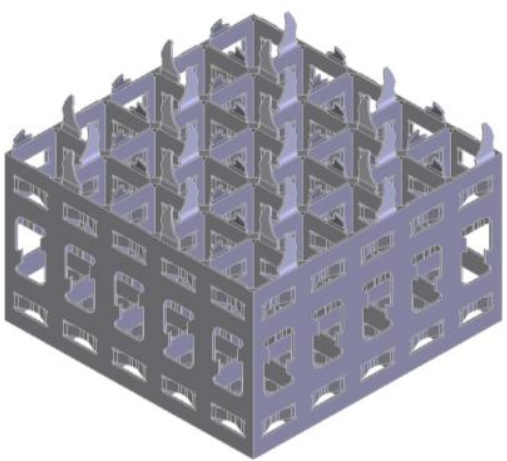

FIGURE 3. $5 \times 5$ subsection of the grid used for experimentation.

The test facility consisted of four grid spacers distributed along the rod's height. The spacer grids had an axial distance between grids of $510.54 \mathrm{~mm}$. This is referred to as the spacer pitch and refers to the distance between the same elevation of successive grids such as the grid bottom edge (or the grid center as shown in Figure 4). It should be noted that the last spacer had a different spacer pitch of $254 \mathrm{~mm}$ with respect to the spacer grid located immediate before. This spacer was located near the channel's outlet and after the measurement zone and it also provided strength and stability to the rod arrays shown in figure 4 . The dimensions of the spacer-grid when located inside the envelope plastic channel for the grid are shown in figure 4. It should be noticed that there was a gap between the edge of the grid and the interior wall of the plastic 
channel. This space was denominated as bypass flow and the results will refer to this space with the same name.A summary of the main dimensions of both grids and their respective flow casing is shown in Table 1.

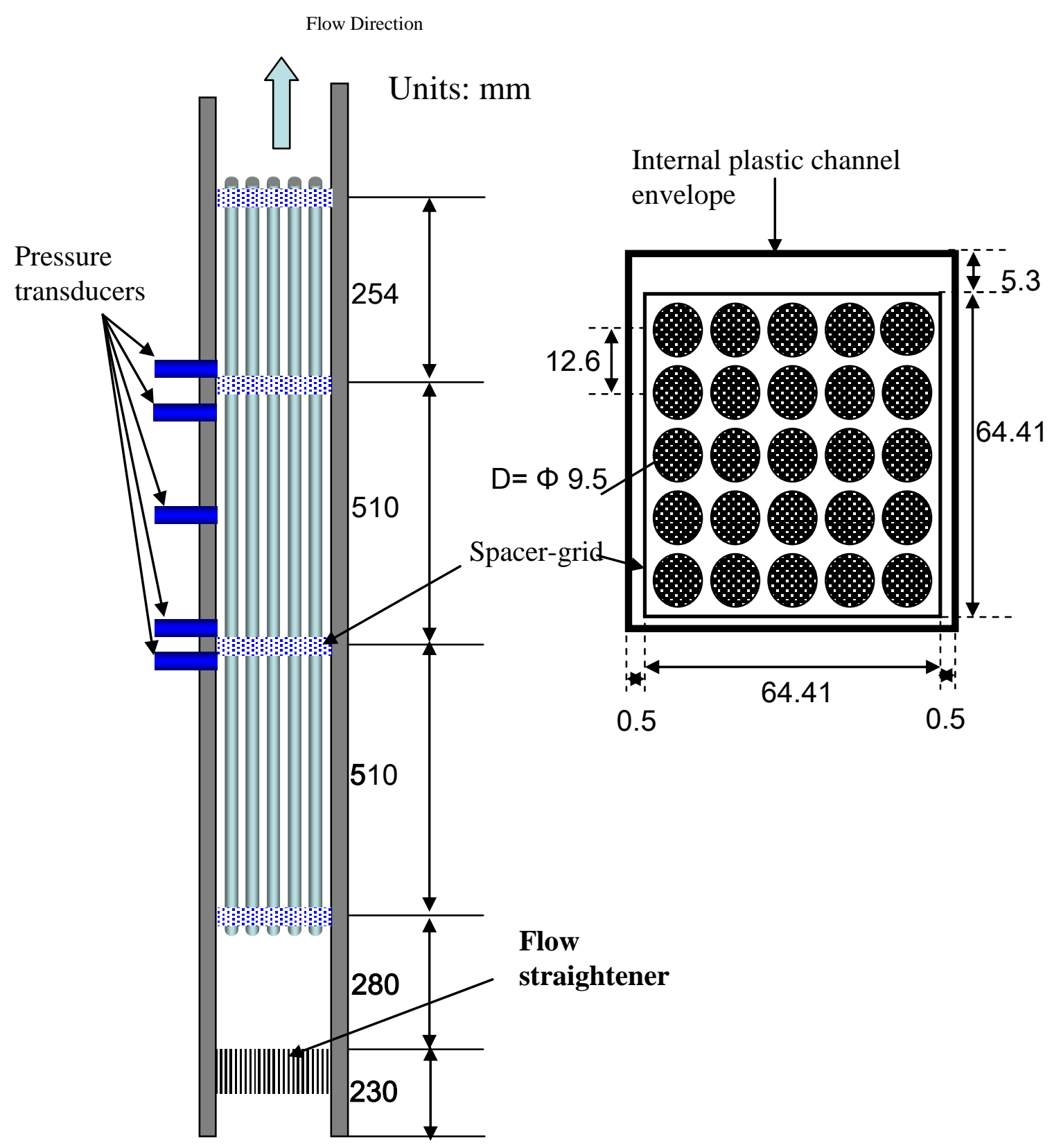

FIGURE 4. Rod bundle with spacer grid and channel envelope dimensions. 


\begin{tabular}{|c|c|c|c|}
\hline & $\begin{array}{c}\text { U-shaped plastic } \\
\text { internal channel } \\
\text { dimensions (mm) }\end{array}$ & $\begin{array}{c}\text { By-passs gap height } \\
(\mathbf{m m})\end{array}$ & $\begin{array}{c}\text { Grid dimensions } \\
(\mathbf{m m})\end{array}$ \\
\hline & & & \\
\hline & & & \\
\hline
\end{tabular}

\begin{tabular}{|c|c|c|c|c|}
\hline & Pitch (P) (mm) & $\begin{array}{c}\text { Average rod } \\
\text { outside } \\
\text { diameter }(\mathbf{m m})\end{array}$ & $\begin{array}{c}\text { Hydraulic } \\
\text { diameter }(\mathbf{D h}) \\
\text { of subchannel } \\
\text { between 4 rods }\end{array}$ & $\begin{array}{c}\text { Distance } \\
\text { between spacers } \\
(\mathbf{m m})\end{array}$ \\
\hline Grid & 12.6 & 9.5 & 11.78 & 510 \\
\hline
\end{tabular}

Table 1. Table showing the grid tested and their respective u-shaped plastic internal channel dimensions.

\subsection{Particle Tracking Velocimety and Optical System}

The optical elements of the experiment included a twin high repetition Nd: YAG class IV laser (New Wave/Pegasus PIV), a beam splitter, mirrors, holders and motorized translation stages. The Nd: YAG laser produced the illumination light for the experiments. It has a maximum power output of $10 \mathrm{~mJ}$ per laser $(20 \mathrm{~mJ}$ total) with a wavelength of $527 \mathrm{~nm}$. The light coming from the laser was directed towards a 50\% mirror which allowed passing through $50 \%$ of the laser beam energy and reflected the other 50\% towards a series of $90 \%$ reflection mirrors. This splitter allowed forming two different laser sheets at the test section. The first laser sheet was parallel to the front face of the channel forming a uniform laser illumination volume of 100 $\mathrm{mm}$ high, $200 \mathrm{~mm}$ long and $1 \mathrm{~mm}$ thick. The second illumination volume had a perpendicular orientation with respect to the front channel face. The second illumination volume had similar dimensions as the illumination volume one. The laser sheet volumes were formed by using a set of three cylindrical lenses. Figure 5 shows a schematic of the optical system used for the experiments. The particle detection and vector calculation was performed using an in-house Particle Tracking Velocimetry (PTV) algorithm. The computer code for PTV has been improved over the years and its reliability tested in several occasions (PIV challenge reference). EstradaPerez (2004) showed that the code has an averaged error of 0.1 pixels for particle detection and velocity calculation. The window size used for the present investigation varied according to the data set. However, for most cases the interrogation window was set to 32 x 24 pixels with an overlap of $50 \%$.

The PTV code output is a velocity field for every pair of images tested. The acquisition rate for the experiments was set to $8000 \mathrm{fps}$ (frames per second). The optimal particle displacement for the cases tested was found to be 8 pixels. One of the advantages of using the 
high speed PTV system described in this section is the acquisition of high speed consecutive flow images. Therefore, the optimum particle displacement for the analysis can be selected by the user. For example, for a case with low flow velocity the particles may have an averaged displacement of 2 pixels in two consecutive images which lead to an increase in the particle detection error (Estrada-Perez 2004). For this case the averaged particle displacement can be increased by changing the number of images to skip. Therefore, if 4 images are skipped for the analysis, the averaged displacement between image number one and image number four is 8 pixels. This approach is possible due to the high acquisition rate of the used PTV system.The instantaneous velocity fields obtained by the PTV algorithm was filtered using a statistic filter. The filter used different methods to detect spurious vectors in the field such as 1) deviation from the mean, 2) vector magnitude outliners, and 3) correlation threshold. It is important to notice at this point that the output of the PTV algorithm and the filter is a velocity field with scatter validated vectors. In other words, the vectors are "real vectors" and no interpolation was done for the resulting instantaneous vectors. The calculation of the statistics presented in this work was done with the instantaneous vector fields; therefore no error due to interpolation was added to the resulted statistics. Once that the statistics were calculated using "real vectors" only, the resulted averaged statistics were organized into a grid for calculation of further parameters such as derivatives which require the existence of a structured grid for its computation.

\section{5 Data Acquisition System}

The data acquisition system included two high-speed/ high-resolution cameras, a frequency cutter circuit, a pulse generator, trigger circuit, and desktop computers for data storage. The cameras were CMOS type sensor Phantom 7.1 and Phantom 7.3 (Vision research). These cameras have a CMOS sensor of 800 x 600 pixels with 4GB of on-board memory and 12 bit pixel depth. The cameras were synchronized with the laser at various frame rates. For frame rates less than $4000 \mathrm{fps}$, the camera was configured to provide the pertinent digital pulses needed to control the laser. Therefore no additional stages were needed for frame rates of up to $4000 \mathrm{fps}$. However for acquisition frequencies exceeding the former limit, a frequency cutter circuit was necessary to assure the correct synchronization of the cameras with the laser shots. The frequency cutter circuit consisted of a pair of type D flip-flops with its clock inputs interconnected in order to cut the frequency of the incoming pulses to half the original value, more details can be found in (Dominguez-Ontiveros et al. 2006). A high accuracy pulse generator (Stanford research) was connected to the output of the frequency cutter circuit to generate the laser trigger pulses. The pulse generator has the capability to receive a pulse and generate an independent pulse with the programmed time delay for each of its outputs. In this work, two channels were used (one per laser) with a programmed time delay depending on the frame acquisition rate (typically $125 \mu \mathrm{s}$ ). The CMOS cameras were used in a master-slave configuration. In this configuration, the master camera generates the pulses necessary to synchronize the image acquisition of both cameras. The frame rate set in the master camera dictate the maximum acquisition rate in order to assure the acquisition of simultaneous frames. The cameras used various optical accessories such as telecentric lenses, filters, ring extenders, in order to capture the desired image resolution and working distances necessary. A second electronic circuit was designed to provide a trigger pulse to the acquisition system. The circuit consisted of a monostable pulse circuit using a 555 Integrated Circuit (IC). The monostable circuit was used to synchronize the picture acquisition and other sensors acquisition (i.e. pressure sensors). 


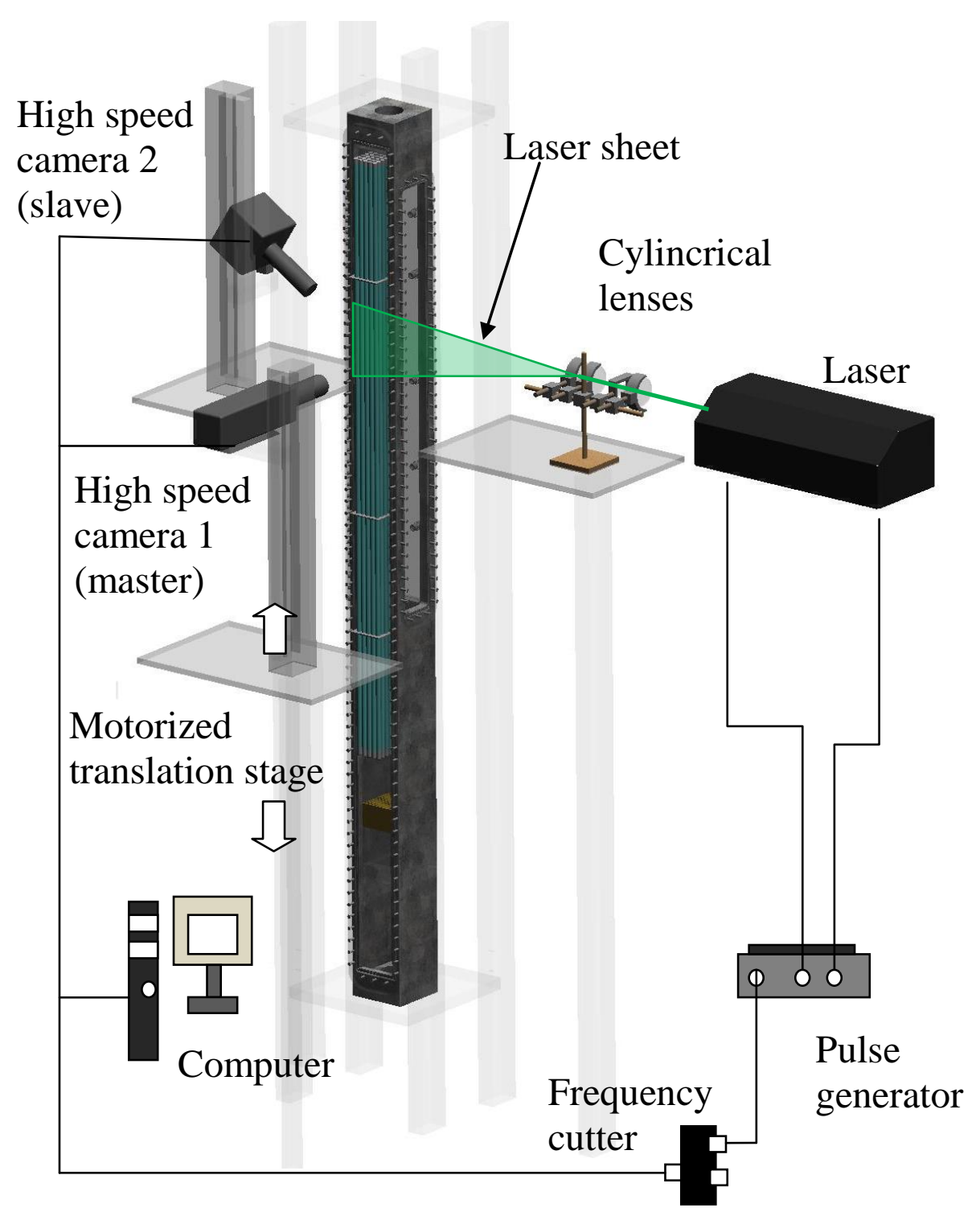

FIGURE 5. Optical system and location with respect to test section. 


\section{Results}

The measurements for axial and lateral velocity at the investigated region were performed at several planes using a multi-scale approach. This approach consisted of performing measurements on various viewing areas of the test section. The scales were selected based on the physical constrictions and the desired resolution of the velocity fields. The measured planes for the tested are shown in figure 6 . Nine planes were measured which are represented by different color lines in the figure. The planes were formed by the laser light where the light passes through the test section. The planes were identified using the number assigned to each of them or by the corresponding notation using the first four letters from the alphabet. For this case plane A was the closest plane to the by-pass flow plane formed by the existent gap between the grid wall and the flow envelope. The first scale examined the flow behavior in the planes that cover the five rods and its respective sub-channels. At this scale the interaction between sub-channels and general characteristics of the flow were captured. The equivalent viewing area of the cameras for this scale was $71.1 \times 52.5 \mathrm{~mm}$ (inter-channel scale). The second scale was selected as the "zoom" to a smaller viewing area in order to increase the resolution of the measurement area (sub-channel scale). This scale captured the flow characteristics of a central sub-channel with an equivalent camera viewing area of $17 \times 12.5 \mathrm{~mm}$. At this scale the flow evolution in the subchannel could be observed.

The planes position was changed using a two-dimensional (Y-Z) positioning system. Figure 6 shows a schematic of the grid and the position of the measured planes. Each plane is represented by a horizontal line with a solid or dashed pattern. The measurements were performed upstream (before grid) and downstream (after grid) of the spacer using the various planes presented in figure 6 .

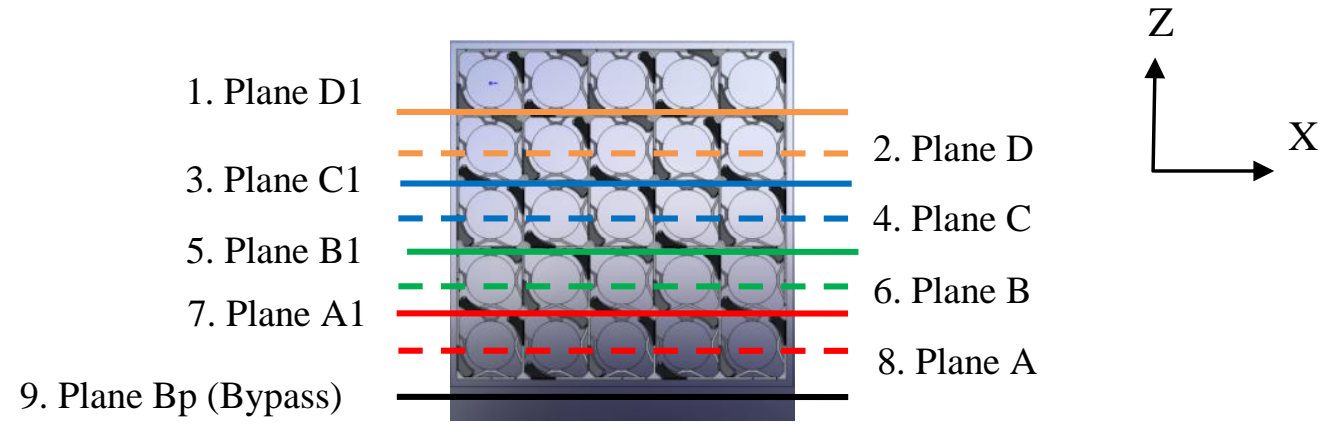

FIGURE 6.Location of the various 2D PIV measurement planes.

In addition, the measurements were performed at several distances from the grid in the upstream and downstream direction. The database includes regions nearby the turbulence promoters attached to the spacer and extend up to 10 hydraulic diameters for each plane in both directions; downstream and upstream the grid. 
The measurements cover the entire $5 \times 5$ rod bundle at the inter-channel scale including the flow after the mixing vanes, the gap between adjacent rods and the wall of the flow envelope. Data was obtained at various Reynolds number ranging from 2000 to 16300 based on the hydraulic diameter of a bundle sub-channel. The obtained velocity fields were used to obtain averaged quantities of the parameters of importance for the investigation. Some of the most important averaged parameters are presented in the following sections. Figure 7 shows the obtained velocity fields for each measured plane upstream and downstream the spacer-grid. The 18 planes measured are compacted into a single plot with the grid position scaled. The rod position was omitted intentionally for better appreciation of the different planes position and its corresponding velocity fields. The white arrow in the middle of Figure 7 represents the main flow direction going from the bottom of the page, passing through the spacer and going upwards. The two positions measured: BGP1 (before the grid, or upstream of the grid) and AGP1 (after the grid, or downstream of the grid) are presented next to its correspondent results. The planes were measured independently at steady state conditions.

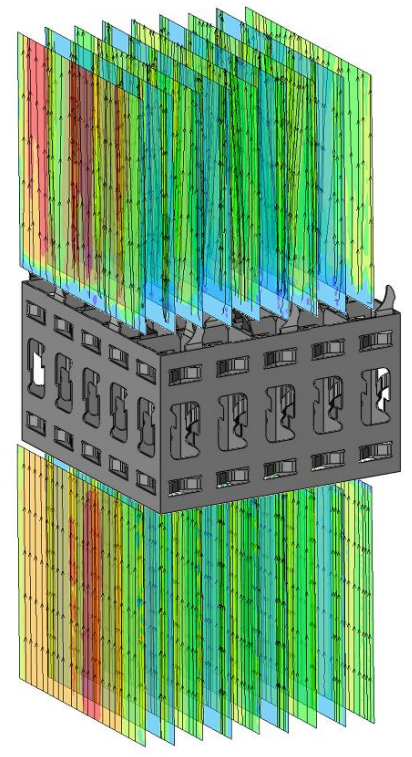

a)
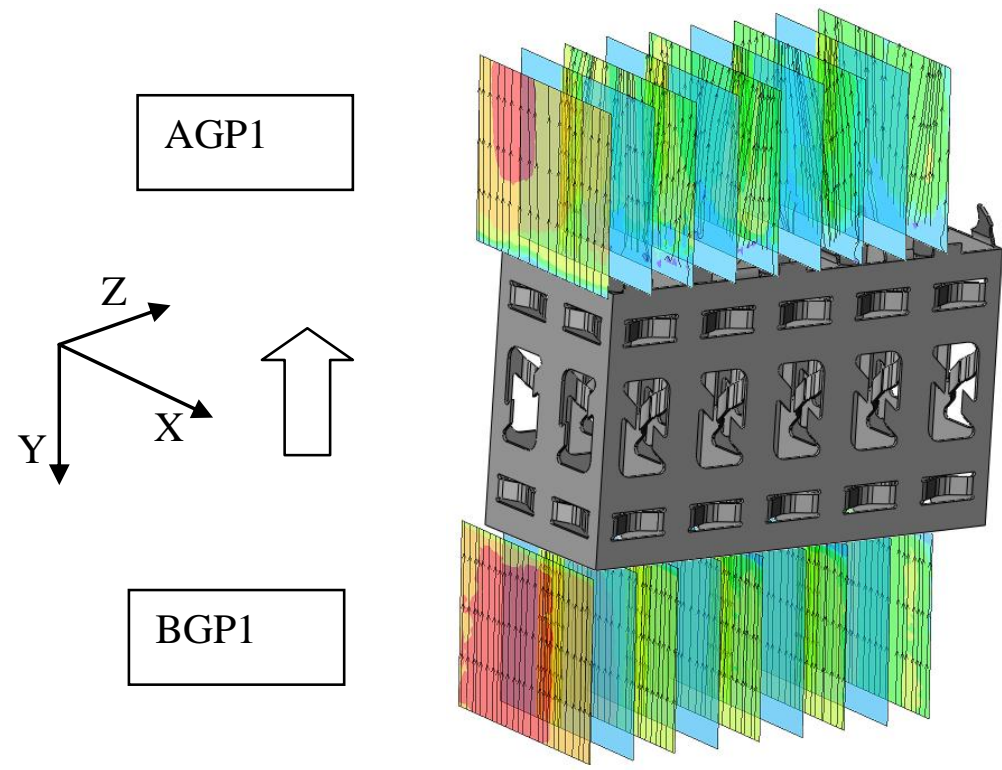

b)

FIGURE 7.Average velocity fields before and after grid. Color code represents streamwise component of velocity vector $(\mathrm{U})$ with red color $=2500 \mathrm{~mm} / \mathrm{s}$ dark blue $=0 \mathrm{~mm} / \mathrm{s}$. $R e=$ 16300. a) Inter-channel scale b) Sub-channel scale

The following results present the velocity profiles of 10,000 images using the assembled averaged process. The average number of vectors per image depends on the plane position. In general, planes that lie between rods had an average of 1000 vectors per image. The planes with no rods had an average of 4000 vectors per image. Therefore, the statistics presented are the result of at least 1 million vectors. It is important to notice that the provided statistics have been performed with vectors that were considered valid vectors during the detection process; however no interpolation was made during the statistical calculations. This step should be noticed since other codes for PIV analysis perform interpolations of the vectors found in order to obtain a 
regular grid of the data. The interpolation process may carry its own disadvantages such as placing vectors where no vectors were present. This issue it is considered important when measurements are performed nearby the spacer-grid or solid boundaries.

The velocity statistics are determined from the PTV measurements by esemble averaging the number of images using equation (1). The turbulence intensities and Reynolds stress quantities were calculated using equartions 2 and 3 respectively (Adrian 2000).

$$
\bar{A}=\frac{\sum_{i=1}^{N} A_{i}(\bar{X})}{N}
$$

where $\mathrm{A}$ is the quantity being averaged, $\overline{\mathrm{X}}$ is the position vector, and $\mathrm{N}$ is the total number of realizations. The result from equation (1) is a two-dimensional average vector field. The intensity of turbulence is defined as the rms value of the fluctuating velocities. The streamwise (u,) and normal (v,) turbulent intensities were calculated using equation 2.

$$
\begin{gathered}
u^{v} \bar{n}(x, y)=\sqrt{\frac{1}{N} \sum_{i=1}^{N}\left[u_{i}(x, y)-U(x, y)\right]^{2}} \\
\overline{u^{\prime} v^{v}}(x, y)=\frac{1}{N} \sum_{i=1}^{N}\left\{\left[u_{i}^{\prime}(x, y)\right]\left[v_{i}^{\prime}(x, y)\right]\right\}
\end{gathered}
$$

Where $u_{i}$ is the instantaneous velocity component in the streamwise direction and $U$ is the mean velocity component in the stramwise direction. The same procedure can be used to obtain the normal component of the velocity vector by substiuing $u^{g}$ for $v^{y}$. Further averaging can be done in the $\mathrm{X}$ or $\mathrm{Y}$ direction when the flow is assumed to be fully-developed. This results in a one-dimensional averaged profile which is a function of $\mathrm{x}$ or $\mathrm{y}$ ( depending on the direction of the averaging) only. For this case, the flow can not be considered as fully-developed since the measurements were made at various zones where the flow is not developed. However, the averaging was performed in a preferencial direction ( $\mathrm{x}$ or $\mathrm{y}$ ) to obtain the 1-D profile for each quantity of interest. The profiles give a simple and efficient way to compare the averaged behaviour of the flow at various measured positions or under different conditions. The following figures show the main averaged turbulence parameters for each plane and each position (upstream and downstream of the spacer) in a one-dimensional profile. The plots for each quantity have been separated into two different plots: 1) planes A, B, C, and D and 2) planes A1, B1, C1, D1 and Bypass for better appreciation of the grid effects. The profiles are color coded and line pattern coded. Similar colors are used for contiguous planes for easy identification in the plot. The line pattern denoting each profile is coded as solid line for all profiles measured at position BGP1 (upstream of the grid) and dotted lines for all profiles measured at position AGP1 (downstream of the grid). 
Figure 8a presents the streamwise component averaged profile for planes with rods under a Re number condition of 16300 . The profiles exhibit a parabolic shape for the central rods upstream the grid. The profiles have a velocity decrease of $30 \%$ from the position upstream of the grid to the same position downstream of the grid. The profiles nearby the containing walls exhibits lower velocities than the central channels. Figure $8 \mathrm{~b}$ shows the profiles for both measured positions under similar conditions for planes without rods. The effect of the bypass flow is denoted by a higher velocity magnitude for the Bp plane. The plane A1, located nearby the Bp plane, shows a higher velocity than the central planes. There is a decrease in velocity for all planes except for the bypass plane for the position AGP1 with respect to the BGP1 measurements. The analysis of the bypass flow profiles is of particular interest in this investigation since the flow is affected by the vanes only and not the grid itself which can be treated as a reduction in cross sectional area. In other words, the flow bypasses the grid, but the zone where AGP1 is located exhibits modification on its velocity profile. The former modification can be only due to the vanes.

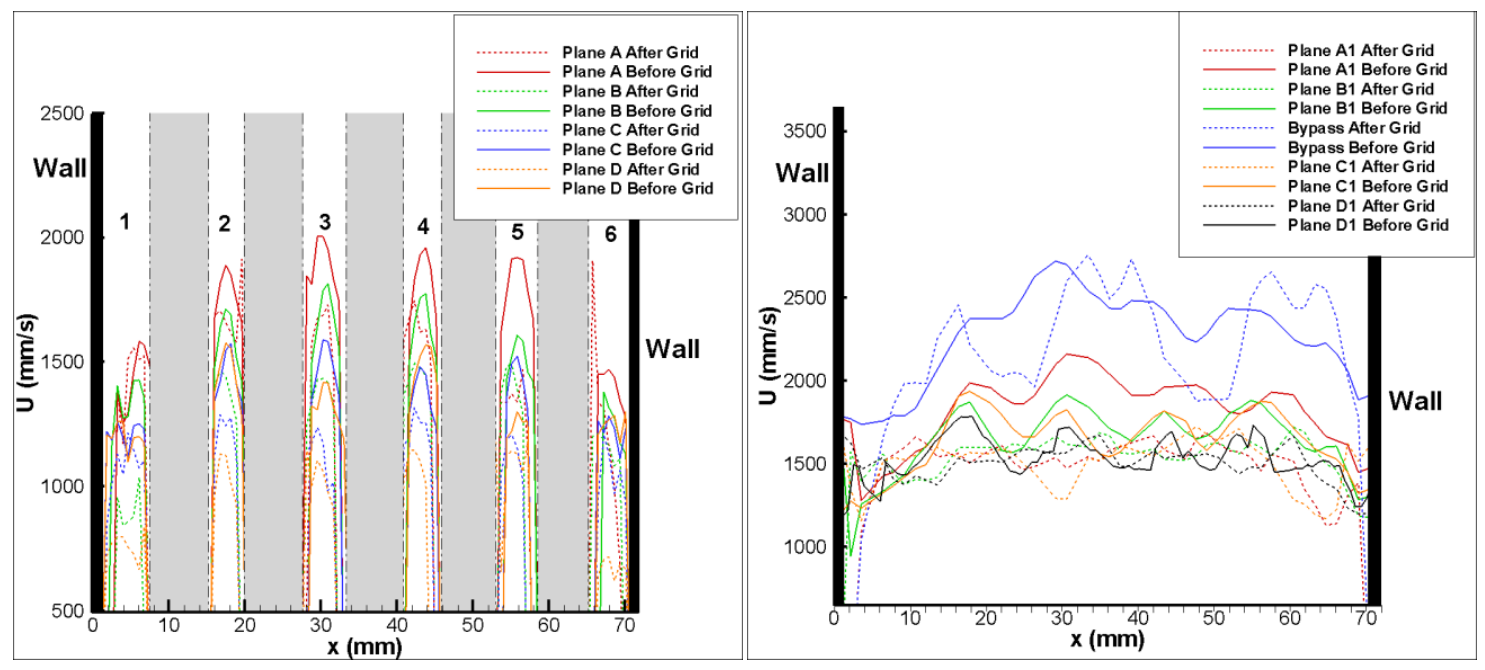

FIGURE 8.Averaged streamwise component of the velocity vector (U). a) Planes $A, B$, and C, D b) Planes A1, B1, C1, D1, Bypass downstream and upstream of the grid. $R e=16300$.

The one-dimensional profiles for the normal (X-axis direction) component of the velocity vector is shown in figure $9 \mathrm{a}$ for the planes with rods and figure $9 \mathrm{~b}$ for planes without rods. The results show mostly a unidirectional change in flow direction for all planes with rods upstream the grid as shown in figure 9. The magnitude of the normal component shows an increase of about $50 \%$ for position AGP1 (downstream of the grid) with respect to position BGP1 (upstream of the grid). Figure 9 show the profiles for both measured positions for the planes without rods. The increase in the normal component at position AGP1 is noticeable with an increment of up to four times the magnitude found at position BGP1. The increment is observed in both directions of the normal component as denoted in the figure by the change in sign. 


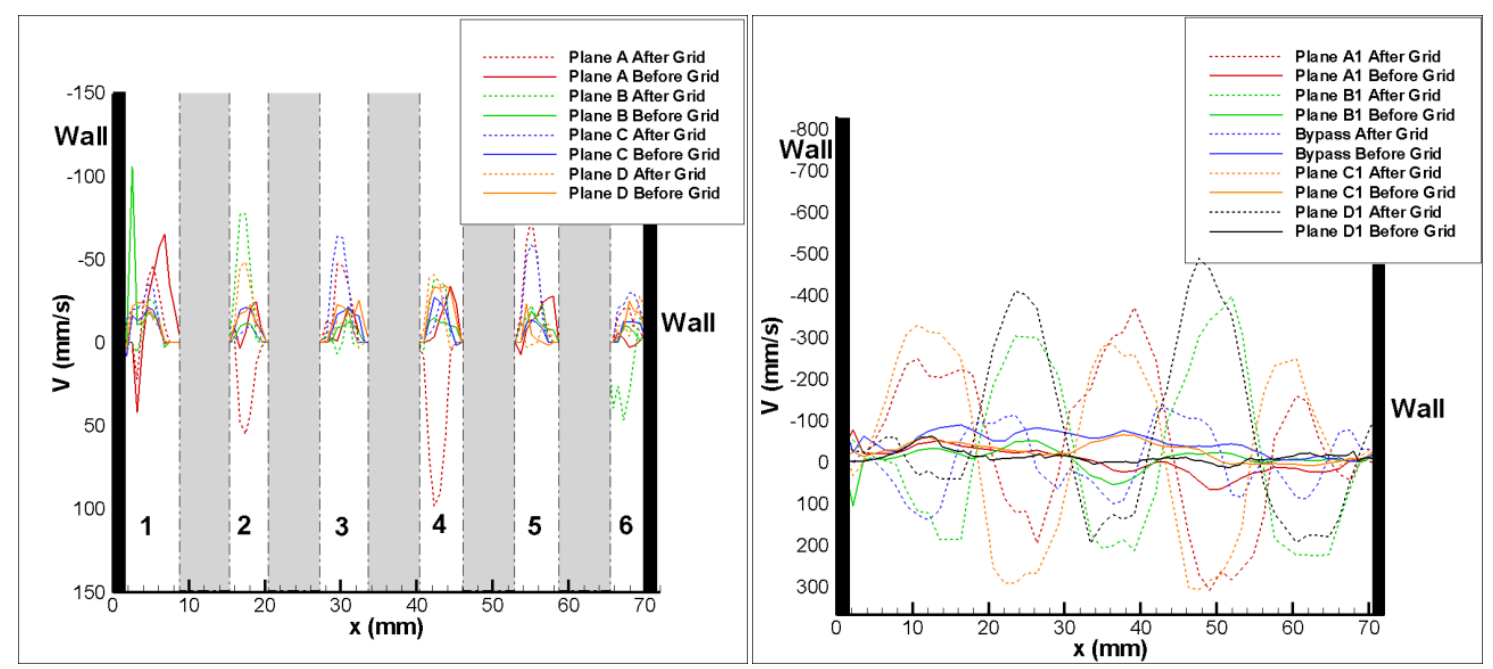

FIGURE 9.Averaged normal ( $\mathrm{X}$-axis direction) component of the velocity vector $(\mathrm{V})$. a) Planes A, B, and C, D b) Palnes A1, B1, C1, D1, Bypass downstream and upstream of the grid. $\operatorname{Re}=16300$.

The turbulence intensities in the streamwise direction $\left(\mathrm{u}_{\mathrm{rms}}\right)$ and normal direction $\left(\mathrm{v}_{\mathrm{rms}}\right)$ of the velocity vector are presented in the following section. Figure 10a shows the streamwise direction velocity fluctuation field for planes with rods. The fluctuations exhibit a behavior similar to the one found in channel flow with solid boundaries where non-slip conditions is enforced for the measured position upstream of the grid. However, this behavior is not persistent downstream of the grid. The fluctuation profile is modified and its magnitude increased by $40 \%$ for position AGP1. The profiles downstream of the grid have a preferential peak direction for several channels caused by the flow change in direction caused by the vanes. The three central channels located in the X-axis at positions $18 \mathrm{~mm}, 30 \mathrm{~mm}$ and $43 \mathrm{~mm}$ have a similar behavior for position BGP1. This fact elucidates that these three channels can be considered as not affected by the containing walls. Figure 10b shows the streamwise turbulence intensities profiles for the planes without rods. The main difference between profiles measured upstream and downstream of the grid is the increase of the intensities by $40 \%$ for the downstream case. Figure 11a shows the averaged profiles for the normal component of the velocity vector for planes with rods. The increment in the turbulence intensities for the normal component at position AGP1 is $40 \%$ higher with respect to the profiles upstream of the grid. The profiles for the normal component corresponding to planes without rods are shown in figure $11 \mathrm{~b}$. For this case, the increment in the normal turbulence intensities is $45 \%$ higher at the downstream position with respect to position BGP1. The normal direction fluctuations for plane Bp (bypass) showed in figure $11 \mathrm{~b}$ exhibit a clear increment (blue dotted and solid lines). The increment indicates the influence of the vanes located at the edge of the grid over the bypass flow. 

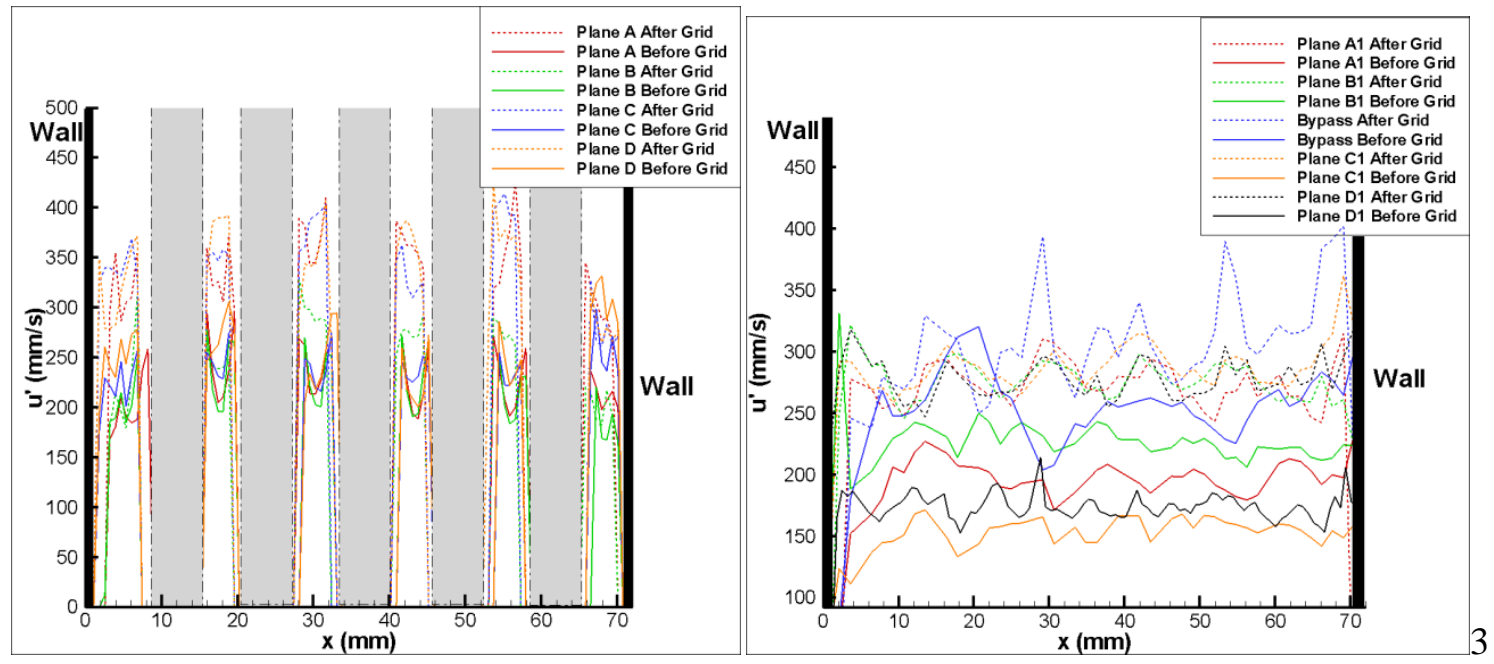

FIGURE 10.Averaged turbulence intensities for the streamwise component of the velocity vector (u'). a) Planes A, B, and C, D b) Planes A1, B1, C1, D1, Bypass downstream and upstream of the grid. $\operatorname{Re}=16300$.

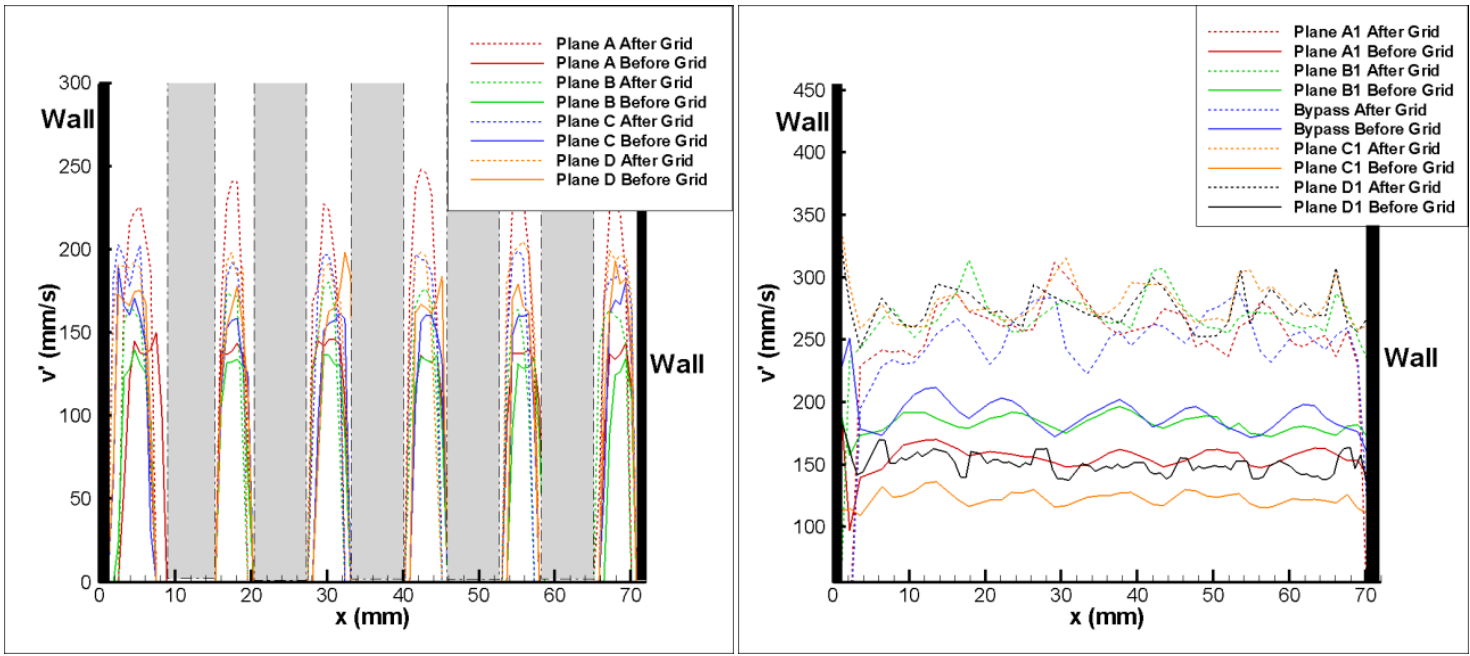

FIGURE 11.Averaged turbulence intensities for the normal component of the velocity vector ( $\left.v^{\prime}\right)$. a) Planes A, B, and C, D b) Planes A1, B1, C1, D1, Bypass downstream and upstream of the grid. $\mathrm{Re}=16300$.

Reynolds stresses in the measured planes, for single phase flow, can be obtained from the velocity fields. The viscous stress can be obtained from the measured streamwise mean velocity profile. Figure 12a shows the averaged Reynolds stresses profiles for planes with rods. The profiles exhibit a similar behavior than the one found in a channel flow with solid boundaries at position BGP1(solid lines in Figure 12a). The stresses are higher near the walls and zero at the center of the channels. In contrast, the stresses show a completely different shape for the position 
downstream of the grid (dotted lines in Figure 12a). Figure 12b shows the Reynolds stresses profiles for the planes without rods. There is an increase in the value of the stress profiles at positions downstream of the grid (dotted lines in Figure 12b) with respect to the position upstream of the grid (solid lines in Figure 12b). This increase in the value of the Reynolds stresses is a function position, as the position increases, the Reynolds stresses decrease. A de-correlation between the streamwise ( $u^{\prime}$ ) and the normal (v') velocity fluctuating components is the source of these decrease of the Reynolds stresses. This phenomenon may be explained due to a modification in the organized structures in the boundary layer.
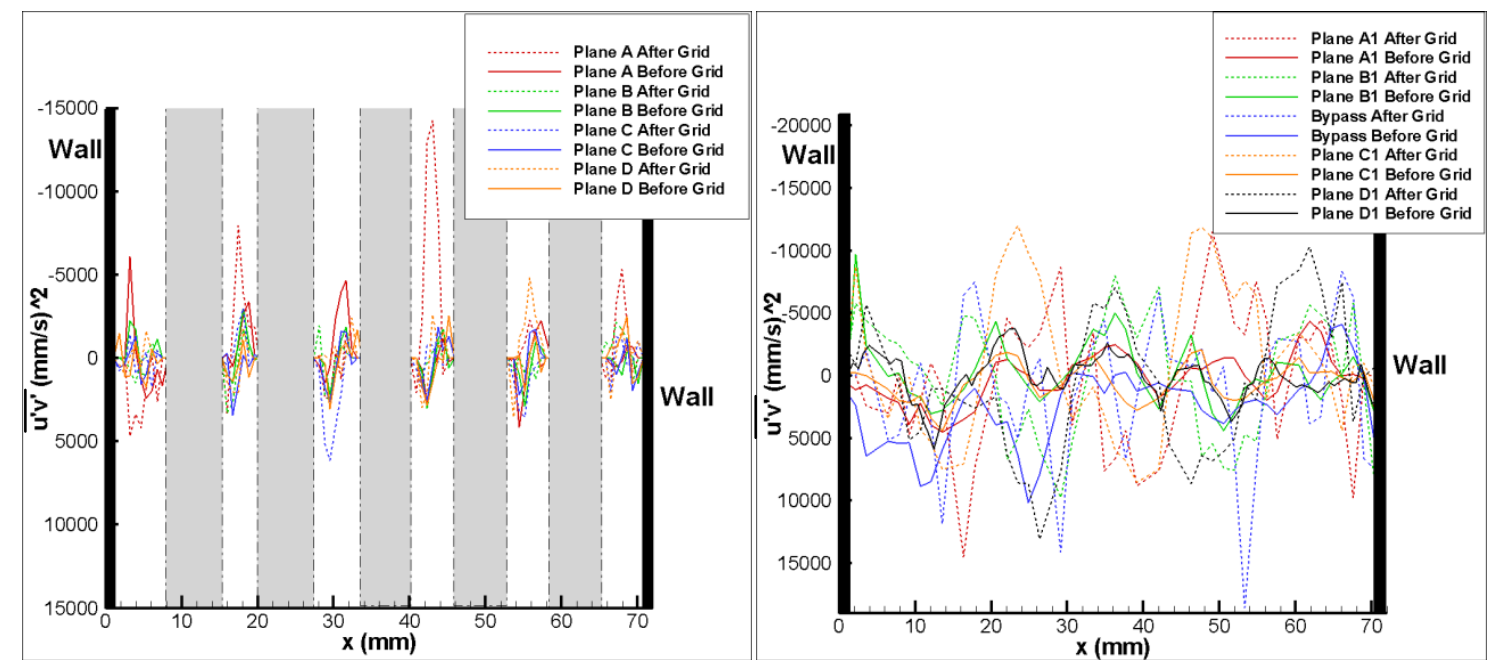

FIGURE 12.Averaged Reynolds stresses (u'v'). a) Planes for the A, B, C, D b) Planes A1, $B 1, C 1, D 1$, Bypass downstream and upstream of the grid. $\operatorname{Re}=16300$.

\section{Conclusions}

Successful use of PIV in addition to Matched Index of Refraction was used for full field velocity fields in a complex geometry. These techniques allowed the measurement of important flow parameters without flow intrusion, eliminating the error due to the interaction of common measuring probes and the flow. The results showed that turbulence evolution can be observed using the TR-PIV technique. Good quality data with high spatial and temporal resolution open the possibility of CFD benchmarking and validation among important quantification of the turbulent structures present in the flow above spacer grids. The experimental results from this work may be useful for the development of a turbulence model for applications such as subchannel geometries, where the flow is highly non-isotropic.

Measurements were performed using a grid design with split type mixing vanes typical of a PWR. The results included the measurement of regions that cover the flow behavior upstream and downstream of the tested grid under various conditions. High quality data was obtained in the vicinity of the grid using the multi-scale approach. Turbulence parameters were calculated from the results obtained from PIV 
The results showed the effect of by-pass flow created by geometrical configurations of the test section. The central sub-channels exhibited no major changes denoting that by-pass flow did not considerably affect the central sub-channels.

The averaged streamwise component of the velocity vector (U) showed a decrease of up to $30 \%$ in the regions immediately above the mixing vanes (downstream) with respect to the results obtained in the regions immediately below the spacer (upstream). The normal component of the velocity vector $(\mathrm{V})$ showed an increase in magnitude for regions near the split vanes of 50 $\%$ due to the inflection caused in flow direction by the split vanes. Turbulence intensities in the streamwise direction ( $u$ ') have an averaged value of $20 \%$ of the maximum velocity $\left(\mathrm{U}_{\max }\right)$ attained in the regions between adjacent rods for measured zones located downstream of the spacer grid. On the other hand, the intensities upstream the grid exhibited an averaged value of $10 \%$ of the maximum velocity.

The averaged normal turbulent intensity (v') had a value of $10 \%$ of maximum velocity $\left(\mathrm{U}_{\max }\right)$ for planes including rods and the planes without the rods. However, the magnitude of the turbulence intensities upstream of the grid, in general, was $40 \%$ smaller with respect to the magnitude of the intensities downstream of the grid.The Reynolds stresses in the gap formed by adjacent rods showed a similar behavior to a channel flow with non-slip condition for measured positions upstream of the grid. This behavior was considerably modified after the flow passed through the grid. The measured regions in the vicinity of the mixing vanes exhibited a considerable change in the behavior of Reynolds stresses.

The turbulence analysis performed on the results obtained showed an increase in the degree of correlation for regions located immediately above the split vanes (downstream) with respect to the regions located immediately below the grid (upstream). The calculated length scales from the correlation fields showed an increase in the length scale of $20 \%$ for regions located upstream of the grid with respect to regions located downstream of the grid. This fact elucidates the modification of eddy size caused by the grid.

\section{Future Work}

One possible outcome of this research is the ability to provide this dataset as an open benchmark. To protect the proprietary nature of the grid design, momentum source terms are being developed. The momentum source terms allows the effect of the grid to be modeled without having to know the exact geometry. These momentum source terms are currently in development. CFD calculations of the tested geometry presented in this work are currently being performed. Comparisons between the experimental data and the CFD calculations under various conditions will be performed. 


\section{References}

ADRIAN, R. J., CHRISTENSEN, K. T. and LIU, Z. 2000 Analysis and interpretation of instantaneous turbulent velocity fields. Eperiments in Fluids 29, 275-290.

CARAGHIAUR, D. and ANGLART, H. 2007 Measurements and CFD predictions of velocity,turbulence intensity and pressure development in BWR fuel rod assembly with spacers. In Proceedings of the 12th International Meeting on Nuclear Reactor Thermal Hydraulics (NURETH12), Pittsburgh, Pennsylvania.

DOMINGUEZ-ONTIVEROS, E. E., ESTRADA-PEREZ, C. E., ORTIZ-VILLAFUERTE, J. and HASSAN, Y. A. 2006 Development of a wall shear stress integral measurement and analysis system for two-phase flow boundary layers. Review of Scientific Instruments 77, 10-32.

ESTRADA-PEREZ, C. 2004 Analysis, comparison and modifcation of various particle image velocimetry (PIV) algorithms. Thesis, Texas A\&M University

IKEDA, K. and HOSHI, M. 2006 Development of rod-embedded fiber LDV to measure velocity in fuel rod bundles. Journal of Nuclear Science and Technology 43, 150-158.

IKENO, T. and KAJISHIMA, T. 2006 Decay of swirling turbulent flow in rod-bundle. Journal of Fluid Science and Technology 1, 36-47.

NETI, S., EICHHORN, R. and HAHN, O. J. 1982 Laser doppler measurements of flow in a rod bundle. Nuclear Engineering and Design 74, 105-116.

REHME, K. 1987 The structure of turbulent flow through rod bundles. Nuclear Engineering and Design 74, 105-154.

ZDRAVKOVICH, M. M. 1987 The effects of interface between circular cylinders in cross flow. Journal of Fluids and Structures 1, 239-261. 